\title{
Hepatocellular carcinoma presenting primarily as ovarian metastasis in Egyptian woman: a case report and literature review
}

\author{
Amal Abd El hafez \\ Pathology Department, Faculty of Medicine, Mansoura University, Egypt \\ E-mail: amalabdelhafez@gmail.com
}

\begin{abstract}
Metastatic spread of hepatocellular carcinoma (HCC) to the ovary is an extremely rare event especially if presented in absence of a known hepatic primary and/or in a living patient. Here, a case of metastatic HCC to the ovary is presented in 54-years living Egyptian female patient in whom the ovarian lesion was the primary presentation associated with elevated serum alpha-fetoprotein level. Ultrasonography revealed 2 two focal hepatic lesions. The diagnosis was based on the simultaneous presence of ovarian and hepatic lesions, bilaterality, the trabecular growth pattern, cytological features, presence of bile and Periodic acid Sciff-positive, diastase-resistant cytoplasmic granules on microscopic examination and immuno-histochemistry. Metastatic HCC must be included in the differential diagnosis of hepatoid and oxyphil cell tumors of the ovary.
\end{abstract}

Keywords: Hepatocellular Carcinoma, Ovarian Metastasis, Hepatoid, Oxyphil, Immuno-histochemistry.

\section{Introduction}

Ovaries are common sites of metastatic tumors and are the most commonly involved organs in the female genital tract (McCluggage and Wilkinson 2005). In order of frequency, the main primary extra-genital sites include; stomach, appendix, colon, breast, small intestine, rectum, gallbladder, urinary bladder and the kidney (Chang et al. 1997, Kiyokawa et al. 2006). However the spread of hepatocellular carcinoma (HCC) to the ovary is extremely rare (Kim 2005, Young 2007, Lee et al. 2011).

The frequency of metastatic tumors that present initially as ovarian masses ranges from $6 \%$ to over $20 \%$ of ovarian metastases in different geographic areas (Seidman et al. 2003, McCluggage and Wilkinson 2005). However, rarely HCC presents as an ovarian metastasis in the absence of a known primary (Young ET al.1992, Pitman et al. 2004).

To the best of our knowledge, few cases of metastatic HCC to the ovary were described in literature, and a small number of such cases have been discovered in living patients, since it was first reported in an autopsy in 1983 (Oortman and Elliott 1983, Young et al.1992, Khunamornpong et al.1999, de Groot et al. 2000, Kim et al. 2004, Kim 2005, Lee et al. 2011).

Because of its broad differential diagnosis (Young 2007); metastatic HCC to the ovary causes diagnostic problems and requires clinical and operative evaluation of the primary tumor (Khunamornpong et al.1999). Here, a case of metastatic HCC to the ovary is reported in a living patient in whom the ovarian lesion was the initial presentation. Based on review of literature, the differential diagnosis and the guidelines for differentiating metastatic HCC to the ovary from its mimics are discussed.

\section{Case presentation}

\section{1. Clinical and imaging findings}

A postmenopausal Egyptian female patient aging 54-years was presented with abdominal distension and bloating. Physical examination disclosed a pelvi-abdominal mass associated with ascites.
Ultrasonography revealed a large left pelvi-abdominal mass, measuring $19 \mathrm{~cm}$. in greatest dimension, most probably ovarian. The right ovary was enlarged and there were 2 two focal lesions in the right hepatic lobe $(9 \times 7 \times 6 \mathrm{~cm}$. and $6 \times 4 \times 2 \mathrm{~cm}$.), with a cirrhotic liver and ascites.

\section{2. Laboratory investigations}

Laboratory investigations evidenced elevated serum glutamicoxaloacetic transaminase (SGOT; $134 \mathrm{IU} / \mathrm{mL}$, normal up to 37 $\mathrm{IU} / \mathrm{mL})$, low serum albumin $(2.9 \mathrm{~g} / \mathrm{dL}$, normal range $3.5-5 \mathrm{~g} / \mathrm{dL})$, and elevated alpha-fetoprotein (AFP; $385 \mathrm{ng} / \mu \mathrm{L}$, normal range, 0 to $7 \mathrm{ng} / \mu \mathrm{L}$ ). The serum was positive for hepatitis B surface antigen, hepatitis Be antigen, and anti-hepatitis B core antibody.

\section{3. Operative procedure}

At laparotomy, there was a left ovarian mass with more than 1500 $\mathrm{mL}$ of hemorrhagic ascitic fluid and carcinomatosis peritonei involving the surfaces of the omentum and the diaphragm. The right ovary was enlarged and contained multiple surface nodules, each measuring up to $1 \mathrm{~cm}$. across. Total abdominal hysterectomy with bilateral salpingo-oophorectomy were performed with drainage of the ascitic fluid. Frozen section examination during surgery showed appearance consistent with metastatic HCC to the ovary.

\section{4. Pathology findings and diagnosis}

Grossly, the left ovarian mass was multinodular, measuring $19 \times 11 \times 9 \mathrm{~cm}$. Predominately solid with a single small cyst $(2 \mathrm{~cm}$. at maximum dimension). The consistency was soft to firm and the cut section showed cream color with a greenish tinge and foci of hemorrhage and necrosis.

Microscopically, hematoxylin and eosin-stained (H\&E) sections from both ovaries showed tumor growth resembling HCC in terms of its architectural and cytological features. The tumor evidenced the typical trabecular pattern, focal pseudo-glandular formations, intercellular canaliculi (Fig. 1a) and bile material (Fig. 1b). Tumor 
cells were polygonal with moderate to abundant pale eosinophilic, granular to clear cytoplasm and vesicular nuclei with one or more prominent nucleoli and mitotic activity. Stromal luteinization, edema of the adjacent ovarian tissue and endothelial bound large spaces were seen (Fig. 1c). Periodic acid Sciff (PAS) stained sections, revealed the presence of numerous PAS-positive, diastaseresistant cytoplasmic granules (Fig. 1d). Cytologic examination of the ascitic fluid revealed cellular samples of polygonal cells having esinophilic cytoplasm arranged in clusters and in trabecular groups (Fig. 2a). The cells showed intranuclear inclusions characteristic of HCC and naked nuclei (Fig. 2b). An applied immuno-

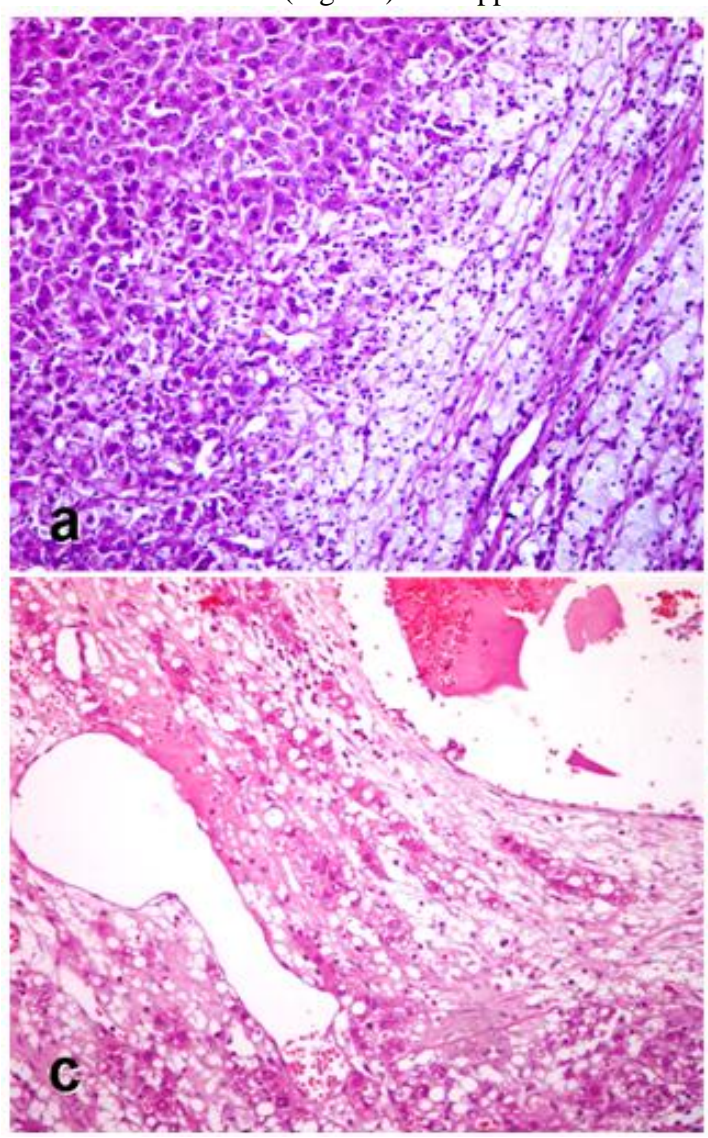

histochemical panel revealed that the tumor was negative for $\mathrm{Cy}$ tokeratin7 (CK7), Calretinin, Inhibin- $\alpha$, GATA-4 excluding the diagnosis of primary ovarian carcinomas, sex-cord stromal tumors and yolk-sac tumor, respectively. Nonetheless, the dual negativity for CK7 and Cytokeratin20 (CK20) was consistent with HHC. Sections prepared from the subsequently resected hepatic focal lesions revealed the same diagnostic histopathologic picture of HHC. The pathological diagnosis of metastatic HCC to the ovary with malignant ascites was confirmed.

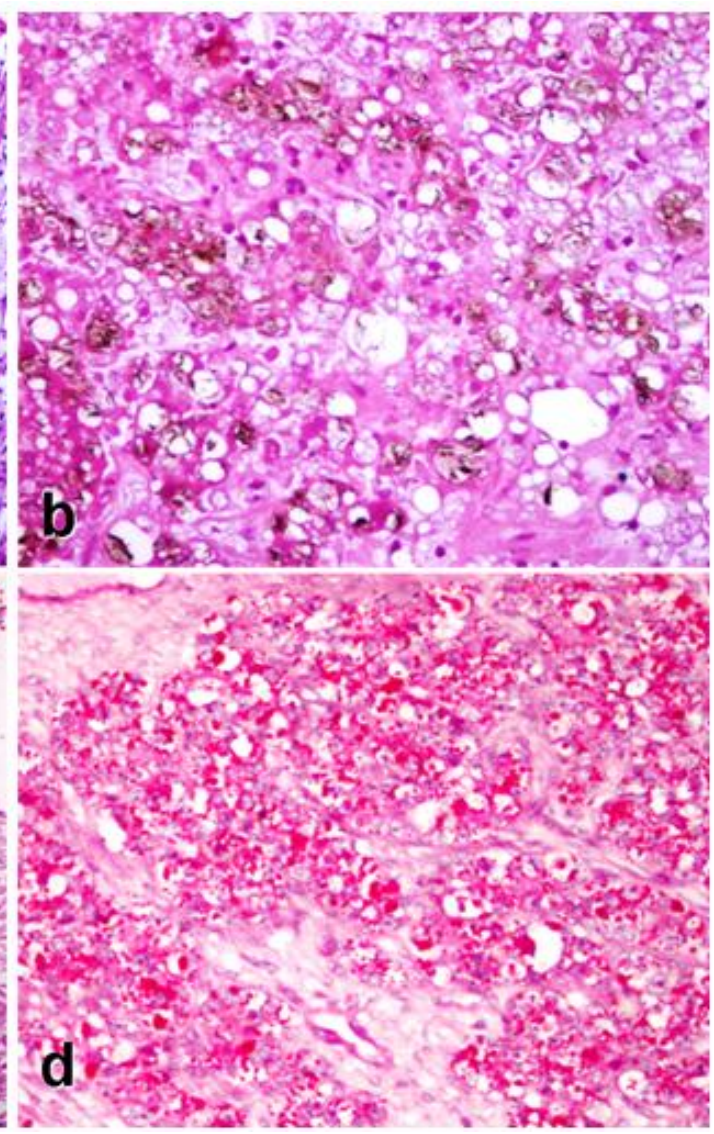

Fig. 1: Metastatic HCC of the Ovary (A) The Tumor Shows Typical Trabecular Pattern, with Intercellular Canaliculi (B) and Bile Material. (C) Ovarian Stromal Luteinization and Edema with Endothelial Bound Large Spaces. (D) PAS-Stained Section Shows Numerous PAS-Positive, Diastase-Resistant Cytoplasmic Granules.

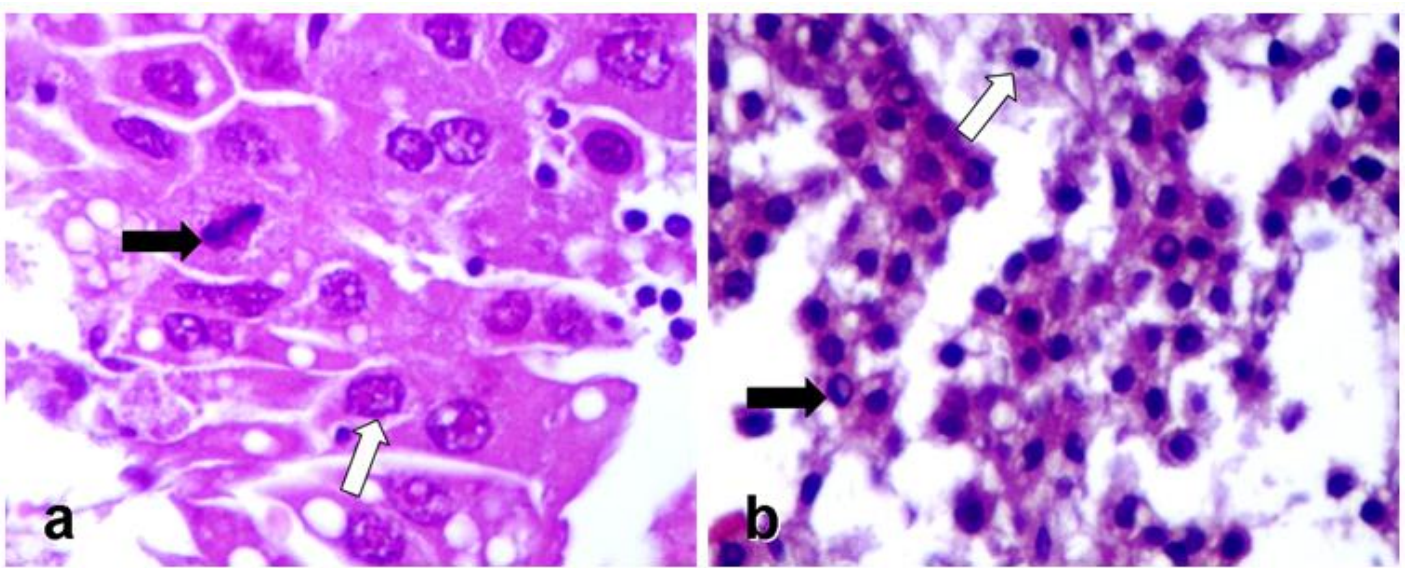

Fig. 2: (A) Cytologic Examination Revealed Clusters and Trabecular Groups of Polygonal Cells Having Eosinophilic Granular to Clear Cytoplasm and Vesicular Nuclei with One or More Prominent Nucleoli (White Arrow) and Mitotic Activity (Black Arrow). (B) There are Naked Nuclei (White Arrow) and Intranuclear Inclusions (Black Arrow). 


\section{Discussion}

Despite the advances in imaging technology, HCC is usually asymptomatic and discovered at advanced stages. The most common sites of metastasis include the lung, peritoneum, adrena gland, and bone, but rarely, the nasal cavity, orbit and gallbladder can be involved (Kim et al. 2004). Nonetheless, HCC metastasizes to the ovary most infrequently (Young 2007), and is often an incidental autopsy finding (Kim 2005).

In most reported cases of metastatic HCC to the ovary, the patient had history of known HCC (Young et al. 1992, de Groot et al 2000, Lee et al. 2011), while the ovarian mass was the initial presentation in some others (Khunamornpong et al. 1999, Kim 2005), as observed in this case. The mean age of patients was 45.6-years (range, 31 to 68 years)(Young et al. 1992, de Groot et al. 2000, Kim 2005, Lee et al. 2011) and nearly all previous reports have shown elevated serum AFP levels as was the situation in the current report. In accordance with Khunamornpong et al. (1999) ovarian involvement was bilateral in this case, but other reports described unilateral ovarian metastases (Kim 2005, Lee et al. 2011). Moreover, these tumors varied widely in size, ranging from $6.5 \mathrm{~cm}$. (Kim 2005, Lee et al. 2011), to $15 \mathrm{~cm}$. in diameter (Khunamornpong et al. 1999), and showed mixed solid and cystic appearance, necrosis and hemorrhage, and sometimes a yellowgreen color which is compatible with bile pigmentation (Khunamornpong et al. 1999, Kim 2005). Yet, the mass presented here had slightly larger dimensions, but with the same gross features as described in literature. In addition, the histopathologic features of this tumor were similar to previously reported cases (Young et al. 1992, Khunamornpong et al. 1999, Kim 2005).The diagnosis of metastatic $\mathrm{HCC}$ in the case presented herein, was based on the simultaneous presence of ovarian and hepatic lesions, elevated serum AFP, bilaterality of the ovarian tumor and the presence of trabecular pattern, bile and PAS-positive, diastaseresistant granules on microscopic examination. Virtually, these features represent the most reliable diagnostic criteria when seen (Kim 2005, Young 2007, Pandey and Truica 2011, Cascales Campos et al. 2013), nonetheless, absence of hepatic involvement should exclude the diagnosis (Gopaldas et al. 2005).

The main differential diagnostic considerations of metastatic HCC to the ovary include tumors with hepatoid differentiation and oxyphil cell tumors (Khunamornpong et al. 1999, Kim et al. 2004, Choudhury et al. 2008). Hepatoid tumors of the ovary are usually associated with elevated serum AFP and comprise two distinct categories: hepatoid yolk sac tumor (HYST) and hepatoid carcinoma either arising from the ovary or metastasizing from a variety of other tissues (Gopaldas et al. 2005), whilst the oxyphil cell tumors of the ovary include oxyphilic clear cell carcinoma (CCC) and steroid cell tumor (Choudhury et al. 2008).

Hepatoid carcinoma is a unique type of carcinoma that arises outside the liver but resembles HCC both histologically and immunohistochemically in its staining for AFP; polyclonal carcinoembryonic antigen; Hepatocyte paraffin 1 (Hep-Par-1) (Pitman et al. 2004), in addition to the presence of PAS-positive, diastase-resistant hyaline globules, meanwhile, bile canalicular structures are not seen in hepatoid carcinoma (Lazaro et al. 2007, Pandey and Truica 2011). These tumors have been reported in the ovary, lung, stomach, renal pelvis, and the bladder (Lazaro et al. 2007). Ovarian hepatoid carcinoma has been described in women with a mean age at diagnosis of 62-years, presenting with unilateral or bilateral ovarian masses (Pandey and Truica 2011). The consistent diagnostic findings of ovarian hepatoid carcinoma are the common admixture with an adenocarcinomatous component of surface epithelial type (usually serous), the diffuse positive staining pattern for Cytokeratin 7 and the elevation of serum CA-125 suggesting an ovarian epithelial origin (Kim MJ 2005, Ulbright 2005, Pandey and Truica 2011).

Hepatoid differentiation was described as scattered clusters of polygonal cells with abundant eosinophilic cytoplasm and prominent nucleoli in $22 \%$ of yolk sac tumors, but rarely, there is a predominant or pure hepatoid pattern in yolk sac tumors (HYST)
(Ulbright 2005). Although HYST is a germ-cell tumor that occurs in younger patients (Pandey and Truica 2011), it may be included in the differential diagnosis of an ovarian tumor with hepatoid differentiation in postmenopausal patients because of its wide range of patients' age (up to54-years) (Khunamornpong et al. 1999). HYST is immunohistochemically positive for AFP and Hep-Par-1 but, detection of foci of more typical yolk sac tumor or other germ cell element, absence of history of long-standing liver disease or tumor in the liver, and positive immuno-staining for GATA-4 can establish the diagnosis of HYST (Khunamornpong et al. 1999, Pitman et al. 2004, Ulbright 2005, Lazaro et al. 2007). Oxyphilic CCC of the ovary is a clear cell carcinoma with a prominent component of cells having abundant eosinophilic cytoplasm. It is an extremely rare neoplasm that occurs between $50-70$ years and is almost always unilateral. Features establishing the diagnosis as a CCC, include tubulocysts lined by cuboidal, hobnail, or flattened cells; papillary structures, nests and sheets of cells with abundant clear cytoplasm containing glycogen; and an adjacent adenofibromatous component (Young and Scully 1987). It presents with elevated CA-125 indicating an ovarian origin of the tumor. Immuno-histochemically, the tumor cells are cytokeratin and epithelial membrane antigen positive and AFP negative (Choudhury et al. 2008).

Ovarian steroid cell tumors, not otherwise specified (NOS), are rare sex cord-stromal tumors of the ovary with malignant potential (Kim et al. 2007), accounting for less than $0.1 \%$ of all ovarian tumors. These tumors may present with amenorrhea and virilization and occur in adults with an average age at diagnosis of 47-years. They can be unilateral or bilateral, sometimes associated with ascites (Jiang et al. 2013). Serum AFP is not elevated with steroid cell tumors, but CA125 is above normal. The tumor forms nodular masses of cells having abundant eosinophilic or foamy cytoplasm (Kim et al. 2007). Inhibin- $\alpha$ and Calretinin are proved to be sensitive and robust immunohistochemical markers in differentiating steroid cell tumors, NOS from non-sex cord stromal tumors (Jiang et al. 2013).

Although metastasis of HCC to the ovary is certainly uncommon, one should have a high index of suspicion in a female patient with a lower abdominal mass and elevated serum AFP. Features, including the age of the patient, laterality of the ovarian tumor, and distribution of disease as evaluated clinically and radiologically, and microscopic features of the neoplasm, establish the diagnosis. Metastatic HCC to the ovary must be included in the differential diagnosis of hepatoid and oxyphil cell tumors.

\section{Disclosure}

No relevant financial affiliations or conflicts of interest to disclose.

\section{References}

[1] McCluggage WG, Wilkinson N (2005) Metastatic neoplasms involving the ovary: a review with an emphasis on morphological and immunohistochemical features. Histopathol 47(3), 231-247.

[2] Chang TC, Changchien CC, Tseng CW, Lai CH, Tseng CJ, Lin SE, Wang CS, Huang KJ, Chou HH, Ma YY, Hsueh S, Eng HL, Fan HA (1997) Retrograde lymphatic spread: a likely route for metastatic ovarian cancers of gastrointestinal origin. Gynecol Oncol 66, 372-377.

[3] Kiyokawa T, Young RH, Scully RE (2006) Krukenberg tumors of the ovary: a clinicopathologic analysis of 120 cases with emphasis on their variable pathologic manifestations. Am J Surg Pathol 30(3), 277 299.

[4] Kim MJ (2005) a case of metastatic hepatocellular carcinoma of the ovary: an immunohistochemical study and literature review. Korean J Pathol 39, 287-290

[5] Young RH (2007) From Krukenberg to today: the ever present problems posed by metastatic tumors in the ovary. Part II. Adv Anat Pathol 14(3), 149- 177.

[6] Lee JM, Park KM, Lee SY Choi J, Hwang DW, Lee YJ (2011) Metastasis of hepatocellular carcinoma to the ovary: a case report and review of the literature. Gut Liver 5(4), 543-547. 
[7] Seidman JD, Kurman RJ, Ronnett BM (2003) Primary and metastatic mucinous adenocarcinomas in the ovaries: incidence in routine practice with a new approach to improve intraoperative diagnosis. Am J Surg Pathol 27(7), 985-993.

[8] Young RH, Gersell DJ, Clement PB, Scully RE (1992) Hepatocellular carcinoma metastatic to the ovary: a report of three cases discovered during life with discussion of the differential diagnosis of hepatoid tumors of the ovary. Hum Pathol 23, 574-580.

[9] Pitman MB, Triratanachat S, Young RH, Oliva E (2004) Hepatocyte paraffin 1 antibody does not distinguish primary ovarian tumors with hepatoid differentiation from metastatic hepatocellular carcinoma. Int J Gynecol Pathol 23(1), 58-64.

[10]Khunamornpong S, Siriaunkgul S, Chunduanb A (1999) Metastatic hepatocellular carcinoma of the ovary. Int J Gynecol\& Obstet 64, 189-191.

[11]de Groot ME, Dukel L, Chadha-Ajwani S, Metselaar HJ, Tilanus HW, Huikeshoven FJ (2000) Massive solitary metastasis of hepatocellular carcinoma in the ovary two years after liver transplantation. Eur $\mathbf{J}$ Obstet Gynecol Reprod Biol 90, 109-111.

[12]Kim TH, Cheung DY, Chung WB, Son DK, Jo DH, Chung JS, Kim HK, Kim JK, Sun HS, Kang CS (2004) A case of metastatic hepatocellular carcinoma of the ovary. Korean J Gastroenterol 43, 215-218.

[13]Oortman EH, Elliott JP (1983) Hepatocellular carcinoma metastatic to the ovary: a case report. Is J Obstet Gynecol 146, 715-717.

[14]Pandey M, Truica C (2011) Hepatoid carcinoma of the ovary. J Clin Oncol 29(15):e446-8. doi: 10.1200/JCO.2010.33.6321.

[15]Cascales Campos PA, Gil Martinez J, Torroba A, Machado F, Parrila Paricio P (2013) peritoneal dissemination of hepatoid carcinoma of the ovary treated with cytoreductive surgery and hyperthermic intraoperative intraperitoneal chemotherapy. Case Rep Med 283295 doi: 10.1155/2013/283295

[16]Gopaldas R, Kunasani R, Plymyer MR, Bloch RS (2005) Hepatoid malignancy of unknown origin: a diagnostic conundrum: review of literature and case report of collision with adenocarcinoma. Surg Oncol 14(1), 11-25

[17]Choudhury M, Pujani M, Singh SK, Biswas R (2008) Oxyphilic clear cell carcinoma ovary. J Postgrad Med 54(4), 326-327.

[18]Lazaro J, Rubio D, Repolles M, Capote L (2007) Hepatoid carcinoma of the ovary and management. Acta Obstet Gynecol Scand 86(4), 498499.

[19]Ulbright TM (2005) Germ cell tumors of the gonads: a selective review emphasizing problems in differential diagnosis, newly appreciated, and controversial issues. Mod Pathol 18 Suppl 2, S61-79.

[20]Young RH, Scully RE (1987) Oxyphilic clear cell carcinoma of the ovary. A report of nine cases. Am J Surg Pathol 11(9), 661-667.

[21]Kim YT, Kim SW, Yoon BS, Kim SH, Kim JH, Kim JW, Cho NH (2007) An ovarian steroid cell tumor causing virilization and massive ascites. Yonsei Med J 48(1), 142-146.

[22] Jiang W, Tao X, Fang F, Zhang S, Xu C (2013) Benign and malignant ovarian steroid cell tumors, not otherwise specified: case studies, comparison, and review of the literature. J Ovarian Res 6(1):53. doi: $10.1186 / 1757-2215-6-53$. 